\title{
Comparison of tumor angiogenesis in subcutaneous and orthotopic LNCaP mouse models using contrast-enhanced ultrasound imaging
}

\author{
Weiyong Liu ${ }^{1}$, Yunkai Zhu' ${ }^{2}$, Lei Ye ${ }^{1}$, Yajuan Zhu ${ }^{1}$, Yuhao Wang ${ }^{3}$ \\ ${ }^{1}$ Division of Life Sciences and Medicine, Department of Ultrasound, The First Affiliated Hospital of USTC, University of Science and Technology \\ of China, Hefei, China; ${ }^{2}$ Department of Ultrasound, Xinhua Hospital Affiliated to Shanghai Jiaotong University, Shanghai, China; ${ }^{3}$ Department of \\ Clinical Medicine, Wannan Medical College, Wuhu, China \\ Contributions: (I) Conception and design: W Liu, Y Zhu; (II) Administrative support: L Ye, Y Zhu; (III) Provision of study materials or patients: W \\ Liu, Y Zhu; (IV) Collection and assembly of data: All authors; (V) Data analysis and interpretation: Y Wang; (VI) Manuscript writing: All authors; (VII) \\ Final approval of manuscript: All authors. \\ Correspondence to: Weiyong Liu. Department of Ultrasound, The First Affiliated Hospital of USTC, Division of Life Sciences and Medicine, \\ University of Science and Technology of China, Hefei 230001, China. Email: lwycsk@163.com.
}

Background: Understanding angiogenesis in prostate cancer is essential. LNCaP prostate xenograft tumors are androgen responsive and closely mimic clinical disease. Orthotopic animal models replicate aspects of the cancer microenvironment and are more clinically relevant than subcutaneous models. Comparative studies investigating angiogenesis using contrast-enhanced ultrasound (CEUS) imaging in subcutaneous and orthotopic mouse models of prostate cancer have not been performed.

Methods: Tumor microcirculation and perfusion in subcutaneous and orthotopic LNCaP xenograft Balb/c athymic nude mice models were compared by investigating microbubble wash-in with CEUS.

Results: The take rate of subcutaneous and orthotopic tumors were $58.3 \%$ and $68.2 \%$, respectively. On CEUS, orthotopic prostate tumors enhanced more rapidly than subcutaneous tumors. Mean arrivaltime (Atm) for subcutaneous tumors, orthotopic prostate tumors, and kidney were 4.21 $\pm 1.86,1.72 \pm 0.79$, and $0.73 \pm 0.12 \mathrm{~s}$, respectively. Mean Atm was significantly longer for subcutaneous tumors compared to orthotopic prostate tumors or kidney $(\mathrm{P}<0.01)$. Mean time to peak enhancement $(\mathrm{TtoPk})$ for subcutaneous tumors, orthotopic prostate tumors, and kidney were $38.56 \pm 13.23,12.39 \pm 7.17$, and $3.74 \pm 1.41 \mathrm{~s}$, respectively. Mean TtoPk were significantly shorter for orthotopic prostate tumors and kidney compared to subcutaneous tumors $(\mathrm{P}<0.01)$. Mean wash-in area under the curve $(\mathrm{WiAuC})$ for subcutaneous tumors, orthotopic prostate tumors, and kidney were $611.11 \pm 247.52,1,800.57 \pm 623.11$, and $1,887.51 \pm 103.68 \mathrm{~dB}$, respectively. Mean AUC was significantly higher for orthotopic prostate tumors and kidney compared to subcutaneous tumors $(\mathrm{P}<0.01)$. Parametric imaging confirmed these findings. The density of CD31-positive vessels was significantly higher in orthotopic prostate tumors $(43.98 \pm 6.14$ vessels/field) compared to subcutaneous tumors $(15.44 \pm 3.74$ vessels/field, $\mathrm{P}<0.01)$.

Conclusions: These findings demonstrate that orthotopic LNCaP xenografts better recreate a proangiogenic microenvironment than subcutaneous LNCaP xenografts.

Keywords: LNCaP; prostate cancer; orthotopic; subcutaneous; contrast-enhanced ultrasound (CEUS)

Submitted Feb 28, 2021. Accepted for publication May 28, 2021.

doi: $10.21037 /$ tcr-21-372

View this article at: https://dx.doi.org/10.21037/tcr-21-372 


\section{Introduction}

Globally, prostate cancer is ranked the most common cancer diagnosed in men (1). Androgen deprivation therapy is widely used in the treatment of localized prostate cancer; however, tumors eventually progress, becoming metastatic and fatal (2). Prostate cancer pre-clinical animal models are being developed to demonstrate the efficacy of novel chemotherapeutic agents (3). This requires the establishment of appropriate criteria to evaluate clinical responsiveness to therapeutic compounds.

Prior studies have shown rich vascularization of orthotopic prostate tumors in rat and PC-3 models. $\mathrm{LNCaP}$ cells are androgen-sensitive human prostatic adenocarcinoma cells. LNCaP cells provide a clinically relevant tool for prostate cancer research (4). $\mathrm{LNCaP}$ xenograft tumors are androgen responsive, which has relevance to human prostate cancer in the clinical setting (5). The LNCaP cell line can be implanted in subcutaneous and orthotopic sites in immunodeficient mice.

Angiogenesis is an important factor in tumor progression and metastasis (6). Contrast-enhanced ultrasound (CEUS) is increasingly applied to visualize and quantify tumor microcirculation (7). In previous studies, dual isotope biodistribution was used to compare vascular perfusion in subcutaneous and orthotopic PC-3 xenograft mouse models (8), nonspecific and target-specific agents were used to investigate early inflow and specific accumulation after orthotopic implantation of G-Dunning rat prostatic tumor cells in Copenhagen rats (9), and a new VEGFR2specific ultrasound contrast agent was used to image rat prostate adenocarcinoma established by injection of G Dunning R-3327 tumor cells in one lobe of the prostate of Copenhagen rats (10). Results showed high vascularization in orthotopic prostate tumors, indicating active angiogenesis.

Reliable animal models of prostate cancer are required to further our understanding of the molecular mechanisms of prostate cancer tumorigenesis. Subcutaneous and orthotopic xenograft animal models are frequently used in medical research. Subcutaneous models are reproducible and tumors become visible and are palpable. Orthotopic models replicate aspects of the cancer microenvironment and are more clinically relevant. Comparative studies investigating angiogenesis using CEUS imaging in subcutaneous and orthotopic mouse models of prostate cancer have not been performed. The objective of the present study is to compare tumor microcirculation and perfusion in subcutaneous and orthotopic LNCaP xenograft mouse models by investigating the time course of microbubble wash-in with CEUS. Results showed that the orthotopic LNCaP xenograft was established as a clinically relevant model of prostate cancer that closely mimics tumor progression and can be monitored using CEUS. We present the following article in accordance with the ARRIVE reporting checklist (available at https://dx.doi.org/10.21037/tcr-21-372).

\section{Methods}

\section{Tumor cell lines}

The LNCaP cell line was obtained from the China Center for Type Culture Collection. LNCaP cells were cultured in Dulbecco's Modified Eagles Medium (DMEM/ F12) (Gibco, USA) supplemented with $10 \%$ fetal bovine serum (FBS, Gibco) in an incubator (HERACELL 150i, Thermo Scientific, Rockford, IL, USA) at $37^{\circ} \mathrm{C}$ in a humid atmosphere containing $5 \% \mathrm{CO}_{2}$. At near $80 \%$ confluence, cells were harvested with $0.25 \%$ trypsin/EDTA and washed twice in PBS. $2.5 \times 10^{6} \mathrm{LNCaP}$ cells in $100 \mu \mathrm{L}$ DMEM/F12 medium were mixed with $100 \mu \mathrm{L}$ Matrigel (BD Biosciences) $(50: 50, \mathrm{v}: \mathrm{v})$ prior to implantation.

\section{Subcutaneous and orthotopic implantation models}

Thirty-six male, 5-week-old Balb/c athymic nude mice were purchased from Shanghai Lab Animal Research Center and acclimated for 2 weeks. Experiments were performed under a project license (No. XHEC-F-2018-050) granted by the Ethics Committee of Xinhua Hospital affiliated to Shanghai Jiao Tong University, School of Medicine, in compliance with institutional guidelines and the National Institute of Health Guidelines for the Care and Use of Laboratory Animals (NIH publication No. 15-8013).

In the subcutaneous model, $200 \mu \mathrm{L} 1 \times 10^{6} \mathrm{~mL} \mathrm{LNCaP}$ cells and Matrigel were subcutaneously transplanted into the left flanks of 12 mice using a 26-gauge needle. From days 30-33 post-injection, tumors were measured each week using a caliper. Tumor volume was calculated as: $\mathrm{V}=0.52 \times$ width $^{2} \times$ length. Mice were sacrificed on day 60 .

In the orthotopic model, $\mathrm{LNCaP}$ cells were grafted into the dorsal prostate of 24 mice. Briefly, mice were anesthetized and placed in a supine position. A $1.5 \mathrm{~cm}$ transverse incision was made in the abdominal wall above the presumed location of the bladder with microscissors. The seminal vesicle was gently pulled outside the 
abdominal cavity using micro-tweezers. The seminal vesicle was stabilized with a cotton ball, and the dorsal prostate was exposed; $20 \mu \mathrm{L} 1 \times 10^{6}$ LNCaP cells and Matrigel were slowly injected into the dorsal prostate using a 33-gauge needle (Hamilton), until a small bubble was observed. The prostate and seminal vesicle were returned to the peritoneum, and the abdominal wall and skin were sutured (5-0 Braided absorbable suture). From the second week after implantation, mice were anesthetized and tumor growth was monitored each week using ultrasound imaging performed with a LOGIQ BOOK-XP system (GE Healthcare, USA) and a $10 \mathrm{~L}$ linear-array probe. The bladder was identified as a round, anechoic structure. The tumor was identified by moving the probe sagittally until an echogenic mass was identified on the dorsal aspect of the bladder. Maximum sagittal length and height of the tumor were measured. The probe was rotated to view the tumor in the transverse plane, and the width of the tumor was measured. Tumor volume was calculated as $0.52 \times$ maximum length $\times$ height $\times$ width, as previously described (11). The stopping criterion for the orthotopic model was a $>20 \%$ body weight reduction.

\section{CEUS and data analysis}

At the end point of the analysis, CEUS was performed with a LOGIQ E9 system (GE Healthcare, USA) and a multi-frequency linear probe (ML 6-15 MHz). Mice were anesthetized and placed in the lateral (subcutaneous) or supine (orthotopic) position according to the method of implantation. The mechanical index was set to 0.08. After visualization of the tumor, $100 \mu \mathrm{L}$ SonoVue ${ }^{\circledR}$ (Bracco, Italy) (diluted in $5 \mathrm{~mL} 0.9 \%$ saline solution after activation) was administered as a bolus via the retro-orbital venous sinus using a $1 \mathrm{~mL}$ syringe (12). Time intensity curve (TIC) analysis from regions of interest (ROI) within the subcutaneous tumors, orthotopic prostate tumors, or kidney was conducted using a 120 s cine loop and LOGIQ E9 built-in quantification software. CEUS-TIC parameters reflecting the hemodynamics of the tissues were derived. Dynamic parameters representative of the wash-in phase after bolus injection of SonoVue were considered, including the arrival-time (Atm), time to peak enhancement (TtoPk), and wash-in area under the curve (WiAuC) (13).

Color-coded parametric images of subcutaneous tumors, orthotopic prostate tumors, and kidney were generated using imaging software incorporated into the GE LOGIQ E9 (14). Parametric imaging monitors microbubble localization by estimating pixel dynamics and shows the detailed hemodynamics of a tissue. The Atm of microbubbles after SonoVue bolus injection was evaluated.

\section{Histological and immunobistochemistry staining}

Tumors were removed and immediately fixed in 10\% formalin and embedded in paraffin. Serial $4 \mu \mathrm{m}$ thick sections were cut on a microtome and mounted on glass slides. For histopathology, standard hematoxylin and eosin staining was performed, sections were incubated with rat monoclonal anti-CD31 (1:500; BD Pharmingen, CA), and secondary antibody was horseradish peroxidase (HRP) conjugated anti-rat IgG (Santa Cruz Biotechnology, CA). Vascular density per area was evaluated at $4 \times$ magnification on six random fields per specimen using Image Pro-Plus software (15) (Media Cybernetics, MD). For immunofluorescence, secondary antibody was antirat Alexa-Fluor 594 (1:2,000; Life Technologies, USA). The tumor nuclei were stained with 4',6-diamidino-2phenylindole, (DAPI) (1:5,000; Santa Cruz Biotechnology, USA). Total number of vessels per visual field was measured at $20 \times$ magnification on six random fields per specimen (16).

\section{Statistical analysis}

Statistical analyses were conducted using SAS 8.02 (SAS Institute, Cary, North Carolina). Continuous data are expressed as means \pm SD. In subcutaneous and orthotopic models, tumor volume, number of vessels stained positive for CD31, and immunofluorescence density were compared using the Wilcoxon rank test, and perfusion parameters (Atm, TtoPk, and WiAuC) were compared using the Mann-Whitney $\mathrm{U}$ test. $\mathrm{P}<0.05$ was considered statistically significant.

\section{Results}

\section{Take rate and monitoring of the subcutaneous and orthotopic models}

In the subcutaneous model, at the end of the study period, all transplanted animals were in good health, and the tumor take rate was $58.3 \%(n=14 / 24)$. The first palpable subcutaneous LNCaP tumors were noted on days 30-33. At this time, tumor color resembled the skin of the nude mice. After 7 days of growth, tumors became gray-black. At the end of the study period, tumors showed a solid and homogeneous morphology. 
A

Day 31

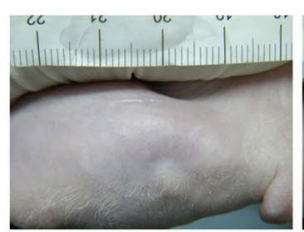

B

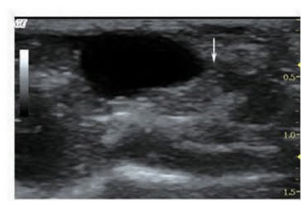

Day 39

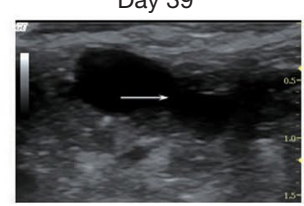

Day 36

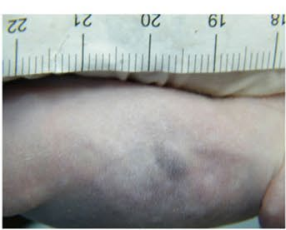

Day 20

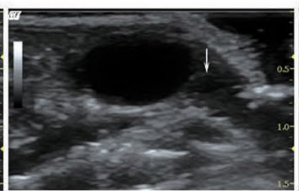

Day 45

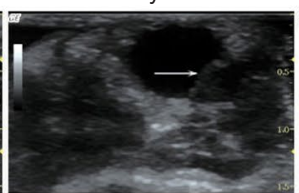

Day 50

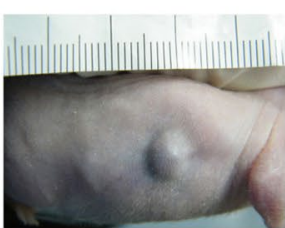

Day 26

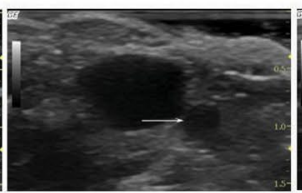

Day 50

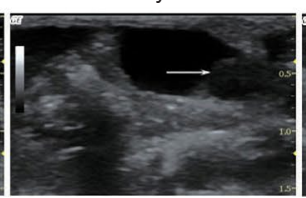

Day 62

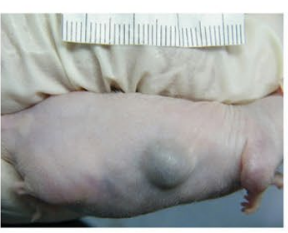

Day 32

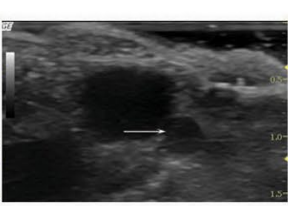

Day 56

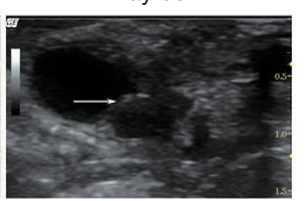

C

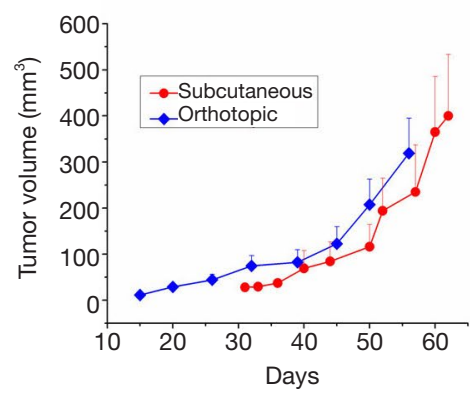

D

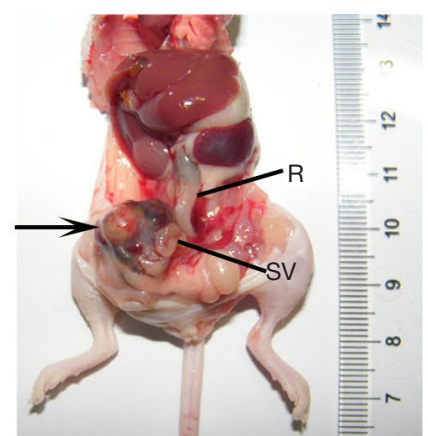

Figure 1 Representative images showing tumor growth. (A) Subcutaneous implantation: After 7 days of growth, subcutaneous tumors became gray-black. (B) Orthotopic implantation: Ultrasound imaging of an orthotopic prostate tumor in the sagittal plane from days 15 to 68. The tumor (white arrow) was located below the bladder and changed from a round hypoechoic structure that impinged on the bladder to an oval hypoechoic structure that compressed the bladder. (C) Tumor growth curves. Subcutaneous tumors were measured each week using a caliper. Tumor volume was calculated as: $\mathrm{V}=0.52 \times$ width $^{2} \times$ length. Orthotopic prostate tumors were measured each week by ultrasound. Tumor volume was calculated as $0.52 \times$ maximum length $\times$ height $\times$ width. (D) Orthotopic prostate tumors (black arrow) formed between the seminal vesicle (SV) and rectum (R).

After orthotopic inoculation, 3 mice died within 2 days due to bowel obstruction. The remaining 22 mice had no postoperative complications. At day 56 postinjection, the tumor take rate was $68.2 \%(\mathrm{n}=15 / 22)$. Orthotopic prostate tumors were discernible on ultrasound on days 15-17 after implantation. At early timepoints, the tumors appeared as hypoechoic, well-defined, rounded structures below the bladder and resembled benign prostatic hyperplasia. The bladder was partly compressed by the tumor. At later timepoints, the tumors became isoechoic, defined, and ovalshaped. Macroscopic observation confirmed the tumors were solid, well-circumscribed, round, dark brown, glossy and located unilaterally in the dorsal prostate (Figure 1).

At day 56, mean orthotopic prostate tumor volume measured by ultrasound $\left(318.81 \pm 76.49 \mathrm{~mm}^{3}\right)$ was significantly greater than mean subcutaneous tumor volume measured by calipers $\left(234.94 \pm 101.99 \mathrm{~mm}^{3}\right)(\mathrm{P}<0.05)$.

\section{CEUS and data analysis}

On CEUS, subcutaneous tumors showed inhomogeneous hypoenhancement and a few scattered strips of enhancement within the tumor. Perfusion defects within the majority of each tumor indicated necrosis.

Orthotopic prostate tumors enhanced more rapidly than subcutaneous tumors. In the initial phase, orthotopic prostate tumors showed more pronounced enhancement towards the periphery than that towards the center, appearing as ring-like features. According to the TICs, mean Atm for subcutaneous tumors, orthotopic prostate tumors, and kidney were $4.21 \pm 1.86,1.72 \pm 0.79$, and $0.73 \pm 0.12 \mathrm{~s}$, respectively. Mean Atm was significantly longer for subcutaneous tumors compared to orthotopic prostate tumors or kidney $(\mathrm{P}<0.01)$, suggesting perfusion differences between tissues. Mean TtoPk for subcutaneous tumors, orthotopic prostate tumors, and kidney were $38.56 \pm 13.23$, 

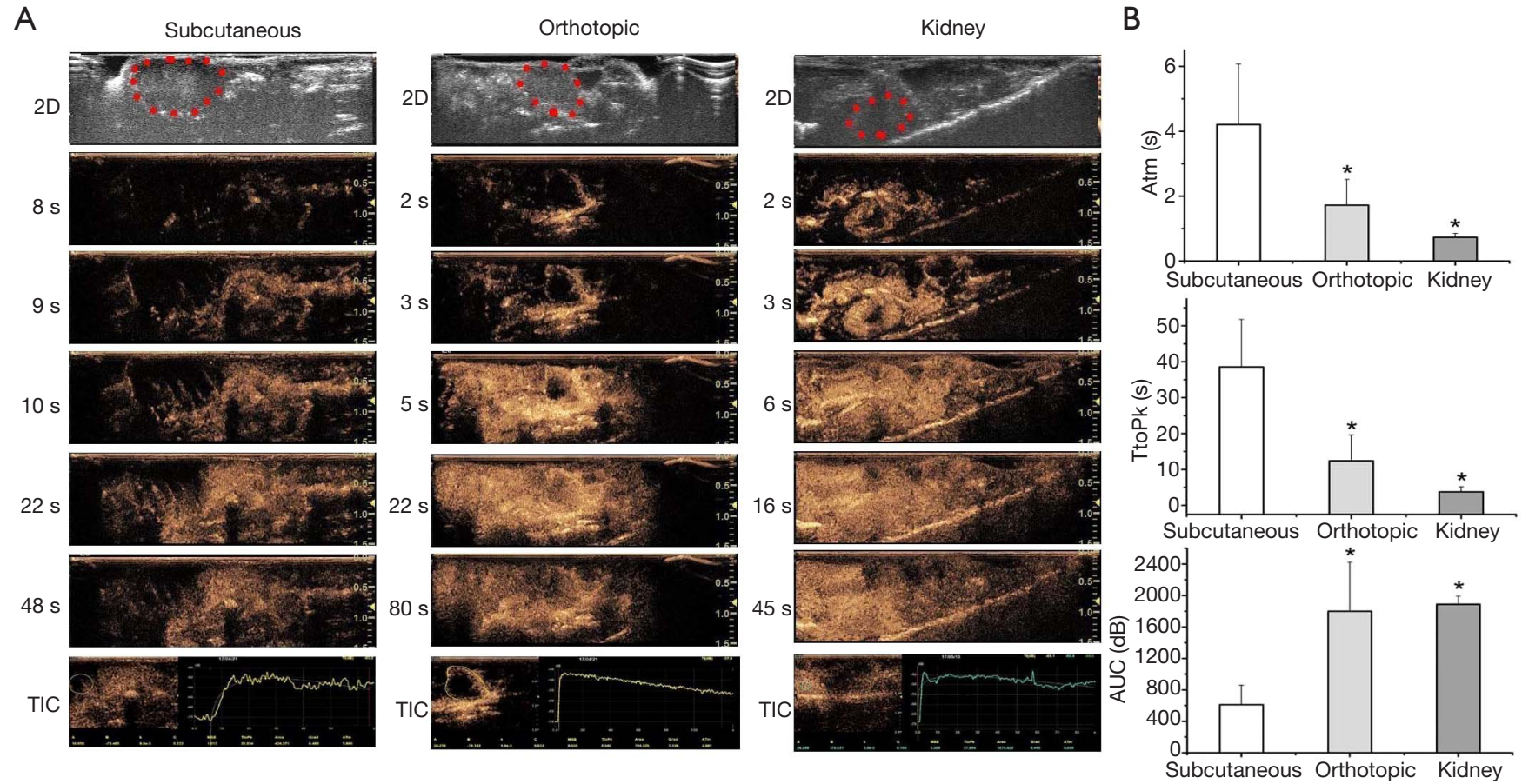

Figure 2 CEUS of a subcutaneous tumor, orthotopic prostate tumor, and kidney (red dashed circles). (A) Contrast enhancement in a subcutaneous tumor, orthotopic prostate tumor, and kidney at various times after injection of SonoVue injection, and corresponding timeintensity curves. There was a steeper increase in signal for both the orthotopic prostate tumor and kidney compared to the subcutaneous tumor. (B) Mean Atm and TtoPk for the orthotopic prostate tumor and kidney were significantly shorter compared to the subcutaneous tumor. The $\mathrm{WiAuC}$ for the orthotopic prostate tumor was 3 -fold higher than the subcutaneous tumor. * denotes statistical significance.

$12.39 \pm 7.17$, and $3.74 \pm 1.41 \mathrm{~s}$, respectively. Mean TtoPk were significantly shorter for orthotopic prostate tumors and kidney compared to subcutaneous tumors $(\mathrm{P}<0.01)$, revealing a rich vasculature in orthotopic prostate tumors. Mean WiAuC for subcutaneous tumors, orthotopic prostate tumors, and kidney were $611.11 \pm 247.52,1,800.57 \pm 623.11$, and $1,887.51 \pm 103.68 \mathrm{~dB}$, respectively. Mean WiAuC was significantly higher for orthotopic prostate tumors and kidney compared to subcutaneous tumors $(\mathrm{P}<0.01)$, indicating the accumulation of microbubbles in the tumor vasculature. Atm, TtoPk, and WiAuC were not significantly different between orthotopic prostate tumors and kidney (Atm: $\mathrm{P}=0.062$; TtoPk: $\mathrm{P}=0.031 ; \mathrm{WiAuC}: \mathrm{P}=0.789$ ) (Figure 2), indicating both orthotopic prostate tumors and kidney had a rich blood supply, and the blood supply of orthotopic prostate tumors could promote tumor growth.

Color-coded parametric images of the subcutaneous tumors showed a slow influx of contrast-agent in the early phase. Orthotopic prostate tumors enhanced from the periphery to the center. The TICs showed no significant difference in mean Atm between the orthotropic prostate tumors and kidney (Figure 2). With parametric imaging, which can capture more detailed information about the progress of tumor enhancement, the orthotopic prostate tumor appeared to enhance slower than kidney (Figure 3).

\section{Histopathological and immunobistochemical characterization of tumors}

Subcutaneous tumors were characterized by massive necrosis and hemorrhage. The morphology of the tumor vasculature was irregular, and vessels had thin walls. Orthotopic prostate tumors were characterized by gross hemorrhage and multifocal necrosis. Orthotopic prostate tumors compressed the prostate gland. Tumor vasculature was abundant and vessels had a large diameter and thickwalls, especially in the peritumoral region. The density of CD31-positive vessels was significantly higher in orthotopic prostate tumors $(43.98 \pm 6.14$ vessels/field) compared to subcutaneous tumors $(15.44 \pm 3.74$ vessels/field, $\mathrm{P}<0.01)$. On immunofluorescence, the number of blood vessels was significantly higher in orthotopic prostate tumors 
A

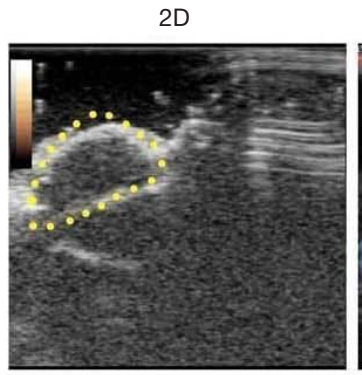

$13 \mathrm{~s}$
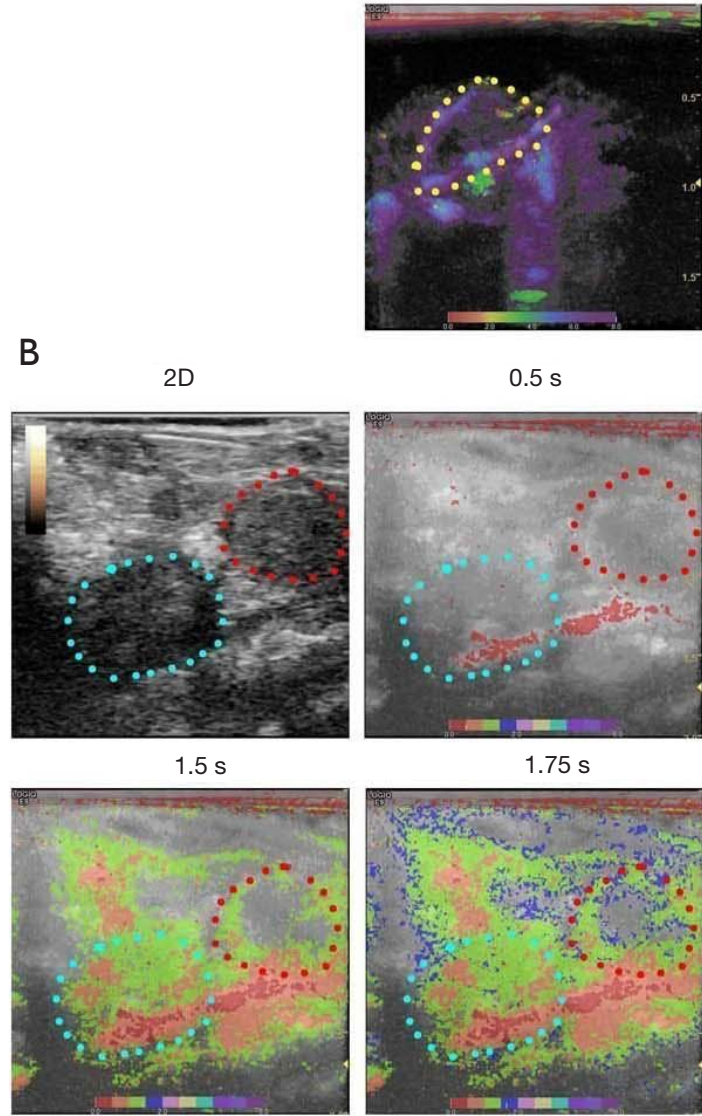

$0.5 \mathrm{~s}$

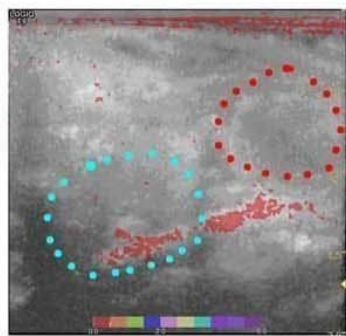

$1.75 \mathrm{~s}$

2D

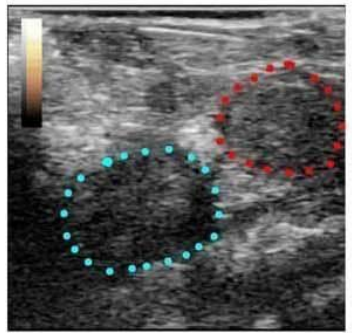

$1.5 \mathrm{~s}$

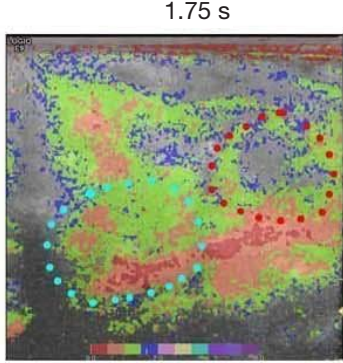

$7 \mathrm{~s}$

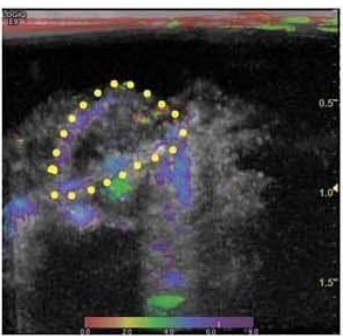

$21 \mathrm{~s}$

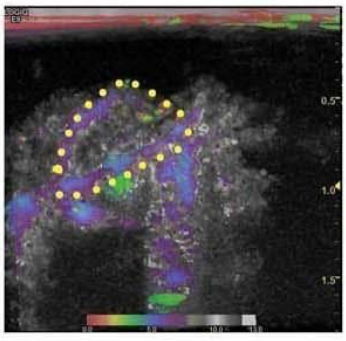

$0.75 \mathrm{~s}$

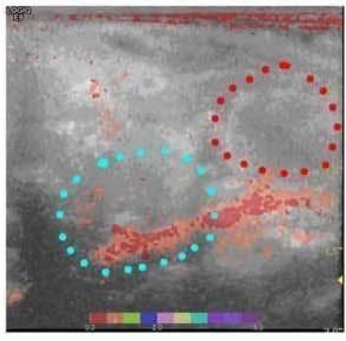

$2.0 \mathrm{~s}$

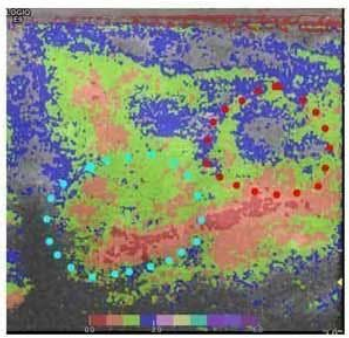

$8 s$

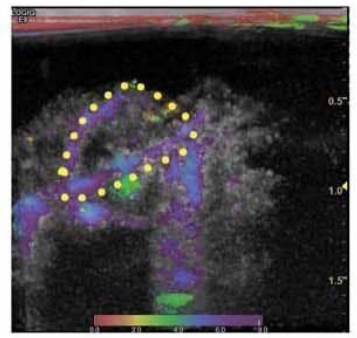

$54 \mathrm{~s}$

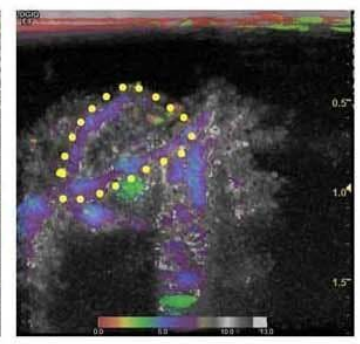

$1.0 \mathrm{~s}$

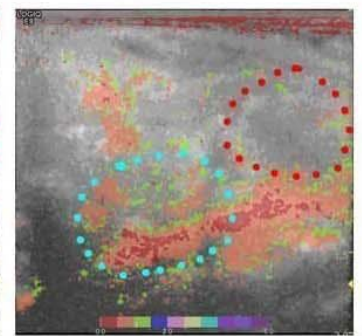

$2.25 \mathrm{~s}$

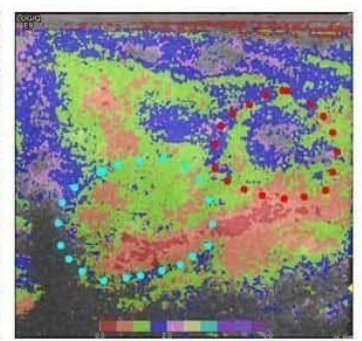

$1.25 \mathrm{~s}$

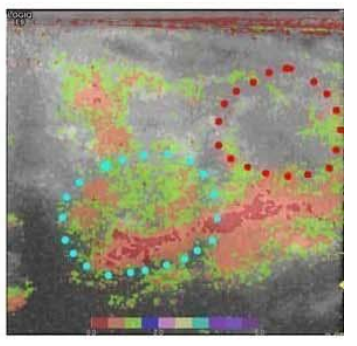

$2.5 \mathrm{~s}$

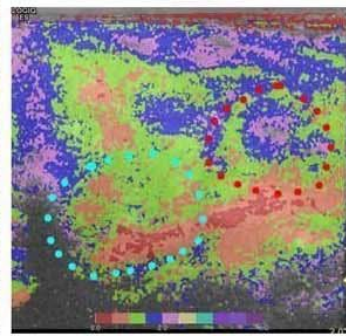

Figure 3 Color-coded parametric images of a subcutaneous tumor, orthotopic prostate tumor, and kidney. (A) Contrast enhancement of the subcutaneous tumor (yellow dashed circles) was slight and slow, and mean Atm was 54 s. (B) Orthotopic prostate tumor (red dashed circles) and kidney (blue dashed circles); green was used to show contrast enhancement. It took $1.5 \mathrm{~s}$ to completely contrast the kidney, by which time only the periphery of the orthotopic prostate tumor was enhanced.

$(52.72 \pm 25.14$ vessels/field) compared to subcutaneous tumors $(24.77 \pm 13.73$ vessels/field, $\mathrm{P}<0.01)$ (Figure 4).

\section{Discussion}

Angiogenesis is involved in progression and metastasis in prostate cancer and is associated with poor survival (17). Reliable animal models of prostate cancer are required to further our understanding of the molecular mechanisms of angiogenesis and develop effective anti-angiogenic therapy (18). This study compared tumor microcirculation and perfusion in subcutaneous and orthotopic $\mathrm{LNCaP}$ xenograft Balb/c athymic nude mouse models by investigating the time course of microbubble wash-in on CEUS. CEUS is an important imaging modality for assessing tumor angiogenesis. CEUS uses microbubbles 
A
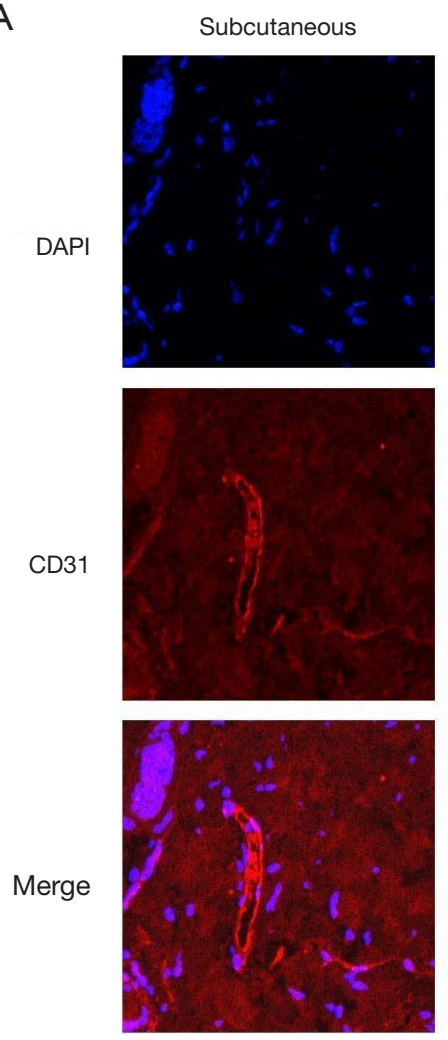

Orthotopic
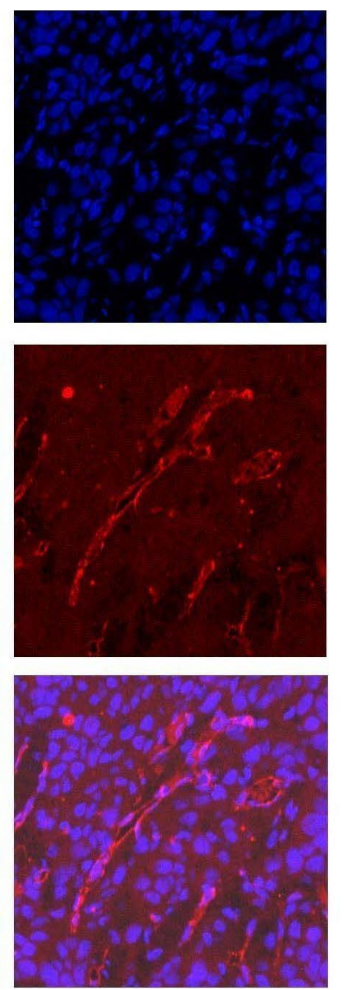

B

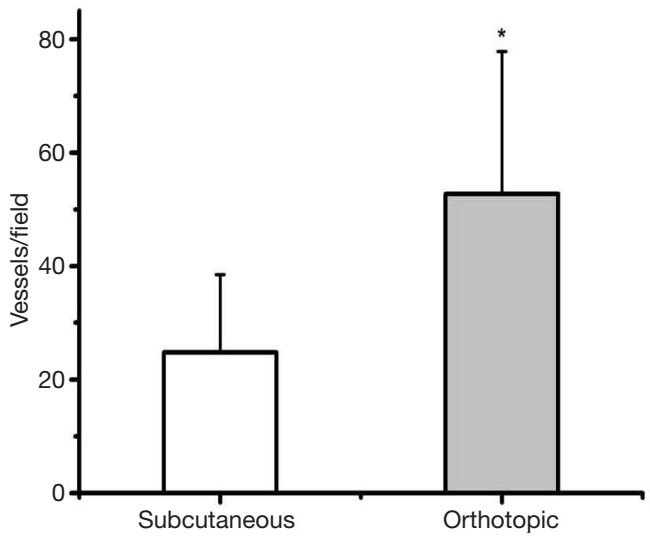

Figure 4 Microscopic observations and immunohistochemistry. (A) Hematoxylin and eosin staining and immunohistochemistry showing CD31 expression levels. In subcutaneous tumors, morphology of the vasculature was irregular and vessels had thin walls and a large diameter. In orthotopic prostate tumors, vasculature was abundant and vessels had thick walls, especially in the peritumoral region. The density of CD31positive vessels was significantly higher in orthotopic prostate tumors; $4 \times$ magnification, mean $\pm \mathrm{SEM}\left({ }^{*} \mathrm{P}<0.05\right)$; (B) on immunofluorescence, blood vessels were stained with CD31 (red) and nuclei were stained with DAPI (blue). There were significantly more blood vessels in orthotopic prostate tumors compared to subcutaneous tumors; $20 \times$ magnification, mean $\pm \mathrm{SEM}\left({ }^{*} \mathrm{P}<0.05\right)$. * denotes statistical significance.

that are infused through a peripheral intravenous catheter to quantify microvascular blood flow and microvascular perfusion. Detecting the features of tumor angiogenesis on CEUS is related to the anatomic characteristics of the tumor, including tumor volume, blood flow, vascular density, and vascular permeability.

Innovative subcutaneous and orthotopic xenografts in immunodeficient nude mouse models have been developed to investigate tumorigenesis in prostate cancer. Primary advantages of subcutaneous xenograft models include ease of implantation and monitoring of tumor growth, while orthotopic xenografts metastasize in a similar manner and to similar locations in rodents as in humans (19). Ultrasound is an optimal imaging modality to monitor orthotopic prostate tumor growth (20). The advantages of ultrasound compared to MRI and bioluminescence include low cost, convenience and real time imaging, and ultrasound allows the clear delineation of orthotopic tumors from the surrounding tissues $(12,21)$. CEUS has been applied in the imaging of tumors in orthotopic prostate cancer xenograft mouse models, showing orthotopic tumors are highly vascularized $(20,22)$. Parametric imaging of contrast ultrasound visualizes hemodynamics on a pixel-by-pixel basis (23). Parametric imaging has been used to differentiate malignant focal liver, breast, and ovarian masses (24).

In the present study, the tumor take rate was facilitated by mixing LNCaP cells with Matrigel (25), and Balb/c nude mice were chosen to facilitate imaging of orthotopic tumors on ultrasound. Findings established the orthotopic LNCaP xenograft Balb/c athymic nude mouse model as a clinically relevant model of prostate cancer that could be used for in vivo testing of novel anti-angiogenic strategies. Consistent with our findings, previous reports show tumor take rates of $50 \%$ and $83 \%$ in subcutaneous and orthotopic 
LNCaP SCID mouse models, respectively, and a tumor take rate of $60 \%$ in an orthotopic $\mathrm{LNCaP}$ athymic mouse model $(26,27)$.

Comprehensive knowledge of angiogenesis in subcutaneous and orthotropic prostate cancer xenograft mouse models and how closely the models resemble human disease is essential, as both models are frequently used in prostate cancer research (28). In the present study, subcutaneous tumors showed lower perfusion than orthotopic prostate tumors. Orthotopic prostate tumors enhanced rapidly from the periphery to the central region, suggesting higher vascularization in peritumoral tissue, which may be indicative of invasiveness and metastases. CEUS-TIC parameters reflecting the hemodynamics of the tumors demonstrated mean Atm and TtoPk were significantly shorter and mean $\mathrm{WiAuC}$ was significantly higher for orthotopic prostate tumors compared to subcutaneous tumors, revealing a rich vasculature and greater persistence of the contrast agent in the tumor vasculature in orthotopic prostate tumors relative to subcutaneous tumors. Consistent with the findings on CEUS, parametric imaging showed orthotopic prostate tumors enhanced faster than subcutaneous tumors.

CD31 is a well-defined marker of angiogenesis that is expressed on the surface of endothelial cells. In accordance with our findings on CEUS, histopathology revealed that the density of CD31-positive vessels was significantly higher in orthotopic prostate tumors compared to subcutaneous tumors. Subcutaneous tumors were characterized by irregular tumor vasculature, and vessels had thin walls. Orthotopic prostate tumors were characterized by abundant tumor vasculature, and vessels had thick-walls and a large diameter, especially in the peritumoral region. On immunofluorescence, there were significantly more blood vessels in orthotopic prostate tumors compared to subcutaneous tumors. These data indicate that the microenvironment at the orthotopic site and cellularenvironmental interaction facilitated tumorigenesis of LNCaP cells (29).

This study was associated with several limitations. First, the number of Balb/c athymic nude mice was small. However, as significant differences in perfusion parameters between orthotopic prostate tumors and subcutaneous tumors were obtained, the use of extra animals was not warranted. Second, CEUS was performed using a clinical transducer rather than a small-animal ultrasound imaging system (30). A small animal ultrasound system with a 30$\mathrm{MHz}$ array transducer may have resulted in ultrasound- triggered microbubble destruction and inaccurate findings. Finally, SonoVue was delivered to the tumors via retroorbital injection instead of tail vein injection to mitigate excessive microbubble destruction. However, some contrast extravasation may have occurred, which could have affected the level of enhancement.

\section{Conclusions}

Findings from the present study imply that CEUS provides an appropriate tool for the assessment of the vasculature in LNCaP orthotopic prostate tumors, and CEUS-TIC parameters and parametric imaging may reflect tumor neovascularity. Applying CEUS to the assessment of angiogenesis in a mouse model of prostate cancer may be helpful for monitoring tumor responses to antiangiogenic therapies and assessing tumor vascularity. Further research should verify the effects and mechanisms of testosterone supplementation on engraftment rates.

\section{Acknowledgments}

The authors thank B. Draper and J. Reynolds for English language editing.

Funding: This work was supported by Major Research and Development Program Projects of Anhui Province (grant 201904a07020095).

\section{Footnote}

Reporting Checklist: The authors have completed the ARRIVE reporting checklist. Available at https://dx.doi. org/10.21037/tcr-21-372

Data Sharing Statement: Available at https://dx.doi. org/10.21037/tcr-21-372

Peer Review File: Available at https://dx.doi.org/10.21037/ tcr-21-372

Conflicts of Interest: All authors have completed the ICMJE uniform disclosure form (available at https://dx.doi. org/10.21037/tcr-21-372). The authors have no conflicts of interest to declare.

Ethical Statement: The authors are accountable for all aspects of the work in ensuring that questions related to the accuracy or integrity of any part of the work are 
appropriately investigated and resolved. Experiments were performed under a project license (No. XHEC-F-2018-050) granted by the Ethics Committee of Xinhua Hospital affiliated to Shanghai Jiao Tong University, School of Medicine, in compliance with institutional guidelines and the National Institute of Health Guidelines for the Care and Use of Laboratory Animals (NIH publication No. 15-8013).

Open Access Statement: This is an Open Access article distributed in accordance with the Creative Commons Attribution-NonCommercial-NoDerivs 4.0 International License (CC BY-NC-ND 4.0), which permits the noncommercial replication and distribution of the article with the strict proviso that no changes or edits are made and the original work is properly cited (including links to both the formal publication through the relevant DOI and the license). See: https://creativecommons.org/licenses/by-nc-nd/4.0/.

\section{References}

1. Siegel RL, Miller KD, Jemal A. Cancer statistics, 2020. CA Cancer J Clin 2020;70:7-30.

2. Karkampouna S, De Filippo MR, Ng CKY, et al. Stroma Transcriptomic and Proteomic Profile of Prostate Cancer Metastasis Xenograft Models Reveals Prognostic Value of Stroma Signatures. Cancers (Basel) 2020;12:3786.

3. Lee HJ, Hwang SI, Byun J, et al. Ultrasound contrastenhanced study as an imaging biomarker for anti-cancer drug treatment: preliminary study with paclitaxel in a xenograft mouse tumor model (secondary publication). Ultrasonography 2017;36:370-7.

4. Liu $\mathrm{W}, \mathrm{Zhu} Y$, Jiang J, et al. Acute hypoxia induces apoptosis in serum-deprived prostate cancer LNCaP cells. Transl Cancer Res 2017;6:1283-93.

5. Grigoryev DN, Long BJ, Njar VC, et al. Pregnenolone stimulates $\mathrm{LNCaP}$ prostate cancer cell growth via the mutated androgen receptor. J Steroid Biochem Mol Biol 2000;75:1-10.

6. Moradi A, Srinivasan S, Clements J, et al. Beyond the biomarker role: prostate-specific antigen (PSA) in the prostate cancer microenvironment. Cancer Metastasis Rev 2019;38:333-46.

7. Turco S, Tardy I, Frinking P, et al. Quantitative ultrasound molecular imaging by modeling the binding kinetics of targeted contrast agent. Phys Med Biol 2017;62:2449-64.

8. Zhang W, Fan W, Rachagani S, et al. Comparative Study of Subcutaneous and Orthotopic Mouse Models of
Prostate Cancer: Vascular Perfusion, Vasculature Density, Hypoxic Burden and BB2r-Targeting Efficacy. Sci Rep 2019;9:11117.

9. Fischer T, Thomas A, Tardy I, et al. Vascular Endothelial Growth Factor Receptor 2-Specific Microbubbles for Molecular Ultrasound Detection of Prostate Cancer in a Rat Model. Invest Radiol 2010;45:675-84.

10. Tardy I, Pochon S, Theraulaz M, et al. Ultrasound molecular imaging of VEGFR2 in a rat prostate tumor model using BR55. Invest Radiol 2010;45:573-8.

11. Saar M, Korbel C, Jung V, et al. Experimental orthotopic prostate tumor in nude mice: techniques for local cell inoculation and three-dimensional ultrasound monitoring. Urol Oncol 2012;30:330-8.

12. Wei S, Fu N, Sun Y, et al. targeted contrast-enhanced ultrasound imaging of angiogenesis in an orthotopic mouse tumor model of renal carcinoma. Ultrasound Med Biol 2014;40:1250-9.

13. Zhou J, Zhang H, Wang H, et al. Early prediction of tumor response to bevacizumab treatment in murine colon cancer models using three-dimensional dynamic contrast-enhanced ultrasound imaging. Angiogenesis 2017;20:547-55.

14. Noro A, Nakamura T, Hirai T, et al. Impact of parametric imaging on contrast-enhanced ultrasound of breast cancer. J Med Ultrason (2001) 2016;43:227-35.

15. Gayed BA, O'Malley KJ, Pilch J, et al. Digoxin inhibits blood vessel density and HIF-1a expression in castrationresistant C4-2 xenograft prostate tumors. Clin Transl Sci 2012;5:39-42.

16. Rohena-Rivera K, Sanchez-Vazquez MM, AponteColon DA, et al. IL-15 regulates migration, invasion, angiogenesis and genes associated with lipid metabolism and inflammation in prostate cancer. PLoS One 2017;12:e0172786.

17. Culp MB, Soerjomataram I, Efstathiou JA, et al. Recent Global Patterns in Prostate Cancer Incidence and Mortality Rates. Eur Urol 2020;77:38-52.

18. Melegh Z, Oltean S. Targeting Angiogenesis in Prostate Cancer. Int J Mol Sci 2019;20:2676.

19. Delitto D, Pham K, Vlada AC, et al. Patient-derived xenograft models for pancreatic adenocarcinoma demonstrate retention of tumor morphology through incorporation of murine stromal elements. Am J Pathol 2015;185:1297-303.

20. Ni J, Cozzi P, Hung TT, et al. Monitoring Prostate Tumor Growth in an Orthotopic Mouse Model Using ThreeDimensional Ultrasound Imaging Technique. Transl 
Oncol 2016;9:41-5.

21. Ravoori MK, Singh S, Yang P, et al. In vivo magnetic resonance imaging of orthotopic prostate cancer. Biotechniques 2020;69:395-403.

22. Kalmuk J, Folaron M, Buchinger J, et al. Multimodal imaging guided preclinical trials of vascular targeting in prostate cancer. Oncotarget 2015;6:24376-92.

23. Slapa RZ, Kasperlik-Zaluska AA, Migda B, et al. Application of parametric ultrasound contrast agent perfusion studies for differentiation of hyperplastic adrenal nodules from adenomas-Initial study. Eur J Radiol 2015;84:1432-5.

24. Korhonen K, Moore R, Lyshchik A, et al. Parametric mapping of contrasted ovarian transvaginal sonography. Ultrasound Q 2015;31:117-23.

25. Jantscheff P, Ziroli V, Esser N, et al. Anti-metastatic effects of liposomal gemcitabine in a human orthotopic LNCaP prostate cancer xenograft model. Clin Exp Metastasis

Cite this article as: Liu W, Zhu Y, Ye L, Zhu Y, Wang Y. Comparison of tumor angiogenesis in subcutaneous and orthotopic LNCaP mouse models using contrast-enhanced ultrasound imaging. Transl Cancer Res 2021;10(7):3268-3277. doi: $10.21037 /$ tcr-21-372
2009;26:981-92.

26. Cunningham D, You $Z$. In vitro and in vivo model systems used in prostate cancer research. J Biol Methods 2015;2:1-28.

27. Wu X, Gong S, Roy-Burman P, et al. Current mouse and cell models in prostate cancer research. Endocr Relat Cancer 2013;20:R155-70.

28. Wang Y, De Leon AC, Perera R, et al. Molecular imaging of orthotopic prostate cancer with nanobubble ultrasound contrast agents targeted to PSMA. Sci Rep 2021;11:4726.

29. Gross NT, Wang J, Fiandalo MV, et al. Recapitulation of prostate tissue cell type-specific transcriptomes by an in vivo primary prostate tissue xenograft model. PLoS One 2020;15:e0233899.

30. Linxweiler J, Korbel C, Muller A, et al. A novel mouse model of human prostate cancer to study intraprostatic tumor growth and the development of lymph node metastases. Prostate 2018;78:664-75. 